Yasukatu Ichigotani • Satoru Matsuda

Kazuya Machida • Kumi Oshima • Takashi Iwamoto

Kenichi Yamaki • Tetsuo Hayakawa

Michinari Hamaguchi

\title{
Molecular cloning of a novel human gene (SIRP-B2) which encodes a new member of the SIRP/SHPS-1 protein family
}

Received: August 11, 2000 / Accepted: September 21, 2000

\begin{abstract}
A full-length cDNA encoding a novel protein was isolated and sequenced from a human placental cDNA library. This cDNA consists of 1735 base pairs and has a predicted open reading frame (ORF) encoding 354 amino acids. It possesses a putative signal sequence, a long extracellular domain, a transmembrane region, a short intracellular domain, and no catalytic domain, which is highly homologous to signal-regulatory protein (SIRP)- $\beta$, suggesting that it seems to be a new member of the SIRP family. Polymerase chain reaction (PCR)-based mapping with both a monochromosomal hybrid panel and radiation hybrid cell panels placed the gene to human chromosome $20 \mathrm{p} 13$ near the marker D20S906.
\end{abstract}

Key words SIRP · SHPS-1 · Glycoprotein · Chromosome 20p13 $\cdot$ RH mapping

\section{Introduction}

Signal-regulatory proteins (SIRPs) comprise several cellsurface glycoproteins (Kharitonenkov et al. 1997). These molecules are structurally characterized by an extracellular Ig superfamily domain, a transmembrane domain, and an

Y. Ichigotani $\cdot$ S. Matsuda $(\bowtie) \cdot$ K. Oshima $\cdot$ M. Hamaguchi Department of Molecular Pathogenesis, Nagoya University School of Medicine, 65 Tsurumai-cho, Showa-ku, Nagoya 466-8550, Japan Tel. +81-52-744-2463; Fax +81-52-735-8167

e-mail: smatsuda@med.nagoya-u.ac.jp

K. Machida $\cdot$ K. Yamaki $\cdot$ T. Hayakawa

Second Department of Internal Medicine, Nagoya University School of Medicine, Nagoya, Japan

T. Iwamoto

Department of Ophthalmology, Nagoya University School of Medicine, Nagoya, Japan

The first two authors contributed equally to this work

The nucleotide sequence data reported in this article have been deposited with the DDBJ, EMBL and GenBank data libraries under the Accession number AB042624. intracellular domain, and are likely to play a significant role in neurons, fibrocytes, and myeloid cells (Fujioka et al. 1996; Kim et al. 1998; Sano et al. 1997; Saginario et al. 1998; Machida et al. 2000). In higher eukaryotes, SIRPs are present as a large group of proteins (Kharitonenkov et al. 1997). However, the physiological role of all of these proteins is not yet clear. The identification and characterization of a variety of proteins in the family are necessary to elucidate the precise mechanisms of the family's cellular function. In this article we describe the cloning and characterization of a novel human gene that encodes a protein presumably belonging to the SIRP family. We named the molecule SIRP-B2, as it seemed to be a new molecule most similar to SIRP- $\beta$.

\section{Methods, results, and discussion}

Rhambda libraries of cDNA were screened by lowstringency hybridization (Kuramochi et al. 1996; Matsuda et al. 1996), using a DNA fragment corresponding to the Src homology 2 containing protein tyrosine phosphatase 2 substrate 1 (SHPS-1) coding domain (amino acid 154-481) as a probe to yield cDNAs encoding full-length clones. One of the libraries used had been produced from poly (A)+RNA isolated from placenta (Clontech, Palo Alto, CA, USA) to get positive clones. DNA sequencing was then performed, using a Sequenase Kit (Amersham, Cleveland, OH, USA) and automatic sequencers for dideoxy sequencing (Amersham, Cleveland, OH, USA) according to the supplier's instructions. The resultant consensus sequence was employed as the correct cDNA sequence. A coding sequence of the positive clone was found to have some homology to the SHPS-1 domain, but was a new gene, suggesting that it represented a potential novel protein belonging to the SIRP/SHPS-1 protein family, as expected. The determined nucleotide sequence and predicted amino acid sequence are shown in Fig. 1A. The cDNA of $1735 \mathrm{bp}$ contains an open reading frame of $1062 \mathrm{bp}$. The SIRP-B2 protein was then predicted to consist of 354 amino acids. 


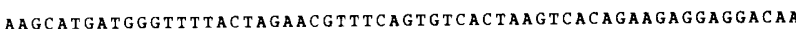
GAAGAGGAATTATGGCAGGGAC CAGCTTTATCACAGTGGTGTGCCTGGTTTGGCAGGGTG CTCACAACTTGTTTGTGGGATGTGGAGGTATCAGAAGTGGAGGTGAGGAGAGCTACAG ATGATTCAGCCTGAGAAGCTCCTGTTGGTCACAGTTGGAAAGACAGCCACTCTGCACTGC \begin{tabular}{lllllllllllllllllllll}
$M$ & $I$ & $Q$ & $P$ & $E$ & $R$ & L & L & L & V & T & V & G & R & T & A & T & L & H & C \\
\hline
\end{tabular} ACTGTGACCTCCCTGCTTCCCGTGGGACCCGTCCTGTGGTTCAGAGGATTGGACCAGGC $\begin{array}{lllllllllllllllllllll}T & V & T & S & \text { L } & \text { L } & P & \text { V } & G & \text { P } & \text { V } & \text { L } & \text { W } & F & \text { R } & G & \text { V } & G & P & G\end{array}$ CGAGATTAATCTACAATCAAAAGAaGGCACTTCCCCAGGTAACAACAGTTCAGAC
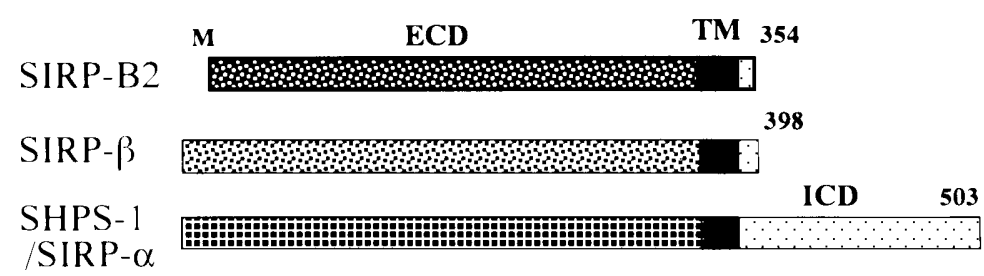

Fig. 1A-C. Representation of the signal regulatory protein (SIRP)-B2 cDNA and sequences. A Nucleotide and predicted amino acid sequences of the $S I R P-B 2$ gene. Numbering of the nucleotide and amino acid sequences is shown on the right. The nucleotide sequence data have been submitted to the GenBank/EMBL data libraries under the Accession number AB042624. The putative signal sequence and transmembrane domain are indicated by thin and thick underlines, respectively. In the 3 '-noncoding DNA sequence, the polyadenylation signal (AATAAA) exists (nt 1713 to 1718). The sequence products were run and analyzed in Amersham Pharmacia, Little Chalfont, Buckinghamshire, UK and a Perkin-Elmer Roche, Branchburg, NJ, USA sequencers, as described in the text. The complete sequence of the cDNA was determined and confirmed by a primer walking strategy, using dideoxy sequencing. B Diagram of schematic protein struc- ture of SIRP-B2, SIRP- $\beta$, and SHPS-1 molecules. The thick hatched and thin dot-patterned open boxes represent the extracellular $(E C D)$ and intracellular (ICD) coding sequences, respectively. The black boxes indicate the transmembrane domain $(T M)$. C Sequence comparison of SIRP-B2 and SIRP- $\beta$. Sequence homology searches were conducted with the protein database at the National Center for Biotechnology Information (National Institute of Health, Bethesda, MD, USA), using the BLASTP and CLUSTALW sequence database search tools. Amino acid sequence comparison between the predicted human SIRP-B2 (amino acids 1-354) and human SIRP- $\beta$ (amino acids $5-398$ ) is shown. Residues of the proteins, indicated by dots (.), represent similar amino acid residues, and gaps are indicated by dashes. The one-letter amino acid code is used 
Fig. 1A-C. Continued

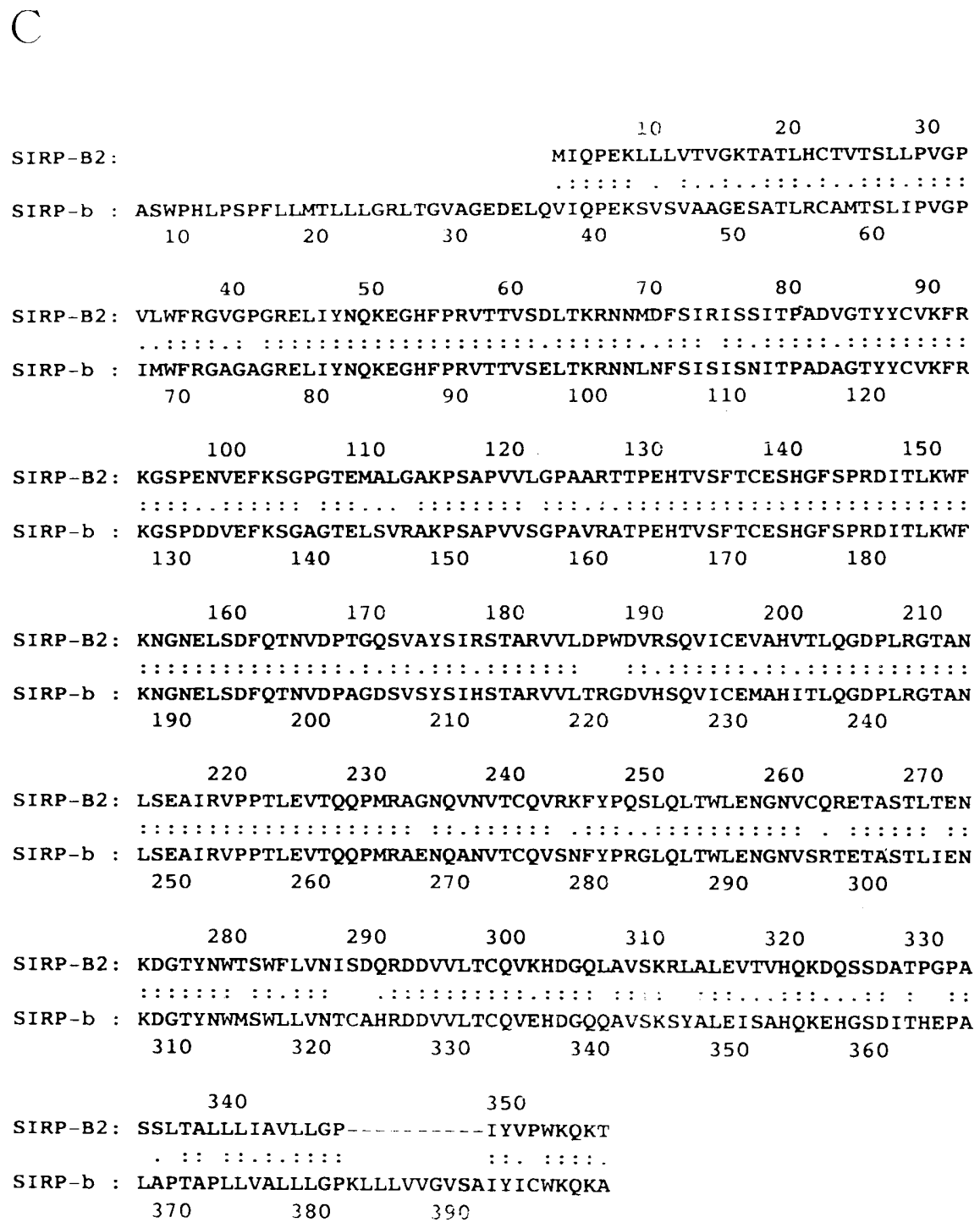

There is a potential ATG start codon favored (Kozak 1989) from position 181 (Fig. 1A). As illustrated schematically in Fig. 1, the predicted SIRP-B2 protein contains a putative signal sequence, a long extracellular domain, a transmembrane region, a short intracellular domain, and no catalytic domain, which is highly homologous to SIRP family members.

A search of the protein database (SWALL and PIR) revealed that SIRP-B2 had high homology to several other proteins. The amino acid sequence of SIRP-B2 was most homologous to that of SIRP- $\beta$ ( $75.5 \%$ identical). The amino acid sequences in the protein were compared (Fig. 1C). However, preliminary analysis indicated that the SIRP-B2 was not a result of alternative splicing (data not shown) (Sano et al. 1999). The deduced amino acid sequence contains a putative transmembrane region that separates an extracellular domain from a cytoplasmic domain that does not include a potential tyrosine-phosphorylation site capable of recruiting some signaling molecules, although SHPS-1 has this site (Veillette et al. 1998). Based on the structural features of SIRP-B2, we propose that SIRP-B2 may also serve as a signal-regulatory receptor for mediating a certain signal transduction. In order to understand the biological functions of SIRP-B2 in more detail, it is important to identify the proteins associated with SIRP-B2. Recently, it has been reported that SIRP- $\beta$ is associated with DAP12, which contains a single cytoplasmic immunoreceptor tyrosine-based activating motif (Dietrich et al. 2000). We speculate that the isolation of these binding proteins to SIRP-B2 may reveal a novel signal transduction pathway.

The tissue distribution of SIRP-B2 transcript in various tissues was examined by using cycle-limited reverse transcription-coupled polymerase chain reaction (RT-PCR), as described previously (Ueki et al. 1999; Seki et al. 1999; Matsuda et al. 2000). Primers used for RT-PCR correspond to the noncoding region of the gene $\left(5^{\prime}>\right.$ AGCTTCTCT AGACTTG $<3^{\prime}$ and $5^{\prime}>$ CCATGAATGAAGACCT $<$ $3^{\prime}$ ); the expected product corresponds to nt 1341 to 1456). Template-cDNAs from the human tissues were purchased from Clontech. As shown in Fig. 2A, SIRP-B2 mRNA expression was detected in brain, heart, lung, liver (most obvi- 

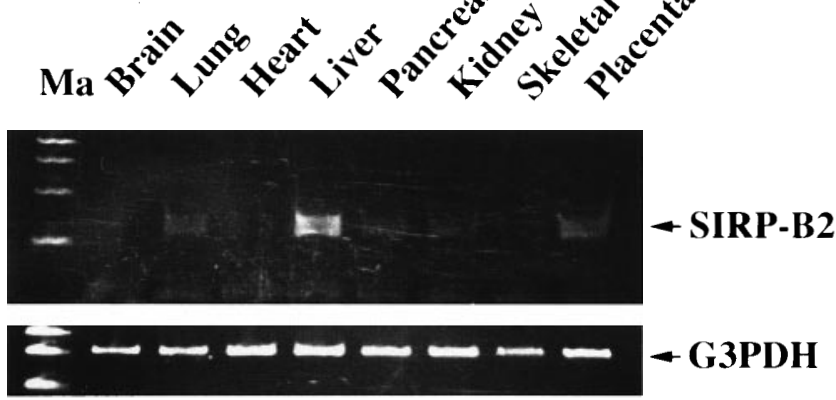

$\begin{array}{llllllll}1 & 2 & 3 & 4 & 5 & 6 & 7 & 8\end{array}$

$\mathrm{B}$

SHPS-1 C B2-1 B2-2

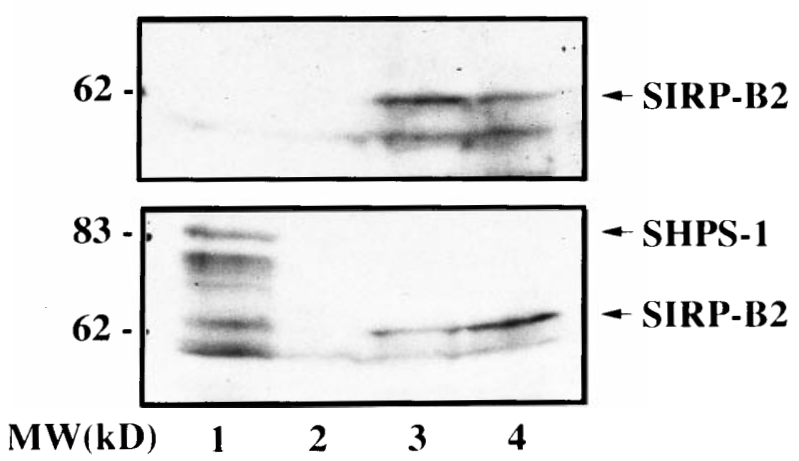

Fig. 2A,B. Expression of $S I R P-B 2$ gene in multiple human normal tissues and identification of the SIRP-B2 protein. A Reverse transcription and amplification by polymerase chain reaction (PCR) with the specific primers for the SIRP-B2 gene were carried out for analyzing $S I R P-B 2$ expression (top panel). The eight tissues examined are indicated above each lane. The template cDNA for brain (lane 1), lung (lane 2), heart (lane 3), liver (lane 4), pancreas (lane 5), kidney (lane 6), skeletal muscle (lane 7), and placenta (lane 8) of the human normal tissues were purchased from Clontech (Palo Alto, CA, USA). Bottom panel shows expression of G3PDH that was analyzed as a control. B Detection of SIRP-B2 protein expressed in COS7 cells. Full-length wild-type GFP-Myc-tagged SIRP-B2 (lanes 3 and 4) and Myc-tagged SHPS-1 gene (lane 1) were reconstituted into pcDNA3.1-plasmid (Invitrogen) under the CMV promoter. These plasmids were then transfected into COS7 cells by lipofection (Lipofect ACE; GIBCO BRL, Grand Island, NY, USA). After $48 \mathrm{~h}$ of transfection, the protein in the lysates was fractionated by $10 \%$ sodium dodecylsulfate-polyacrylamide gel electrophoresis (SDS-PAGE) and immunoblotted with both anti-GFP (top panel) and anti-Myc (bottom panel) antibodies. Lane $2(C)$ represents a non-transfected negative control

ous), pancreas, kidney, placenta, and in skeletal muscle by using cycle-limited RT-PCR (28 cycle-amplified), suggesting that SIRP-B2 may be ubiquitously expressed, although the levels of expression varied. In this experiment, genomic DNA may not have been amplified, because primers designed from intron DNA of $S I R P-B 2$ could not be amplified (data not shown). Considering its expression profile, SIRP$B 2$ may be involved in a basic function of many cells, especially those in liver. To analyze the protein product of $S I R P-B 2$, western analysis was performed. As shown in Fig. 2B, when a cDNA encoding the corresponding GFP-Myc-
A

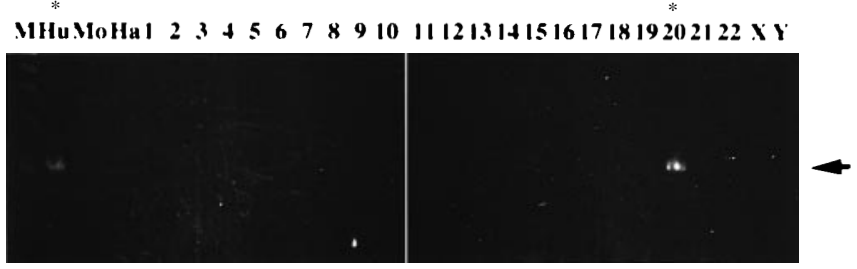

$\mathrm{B}$

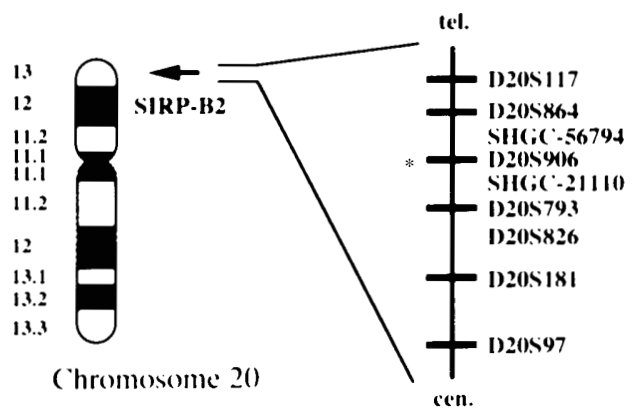

Fig. 3A,B. Chromosomal assignment of the $S I R P-B 2$ gene. A Representative PCR-based monochromosomal somatic cell hybrid mapping of SIRP-B2. Primers from the $3^{\prime}$-untranslated region (UTR) of the SIRP-B2 cDNA were used. Lanes 1-22 and lanes $X$ and $Y$ represent human chromosomes. Lanes $\mathrm{Hu}, \mathrm{Mo}$, and $\mathrm{Ha}$ represent cell hybrids containing genomic DNA from human, mouse, and hamster, respectively. Lane $M$ represents marker. A single product of the expected size was generated from chromosome 20 and human genomic DNA (indicated by asterisk). B Chromosomal localization of the SIRP-B2 gene in schematic ideogram of human chromosome 20. Illustration of the approximate corresponding cytogenetic location of the gene on the chromosome 20 p13 by radiation hybrid (RH) panel mapping is shown. Asterisk shows the putative localization of the SIRP-B2 gene

tagged SIRP-B2 protein was expressed in COS7 cells using pcDNA3.1 expression-plasmid (Invitrogen, Carlsbad, CA, USA), anti-tag (both anti-GFP and anti-Myc) antibodies (MBL) specifically detected a $65-\mathrm{kD}$ a protein in the lysates of transfected COS7 cells (Fig. 2B; lanes 3 and 4). When a cDNA encoding the corresponding Myc-tagged SHPS-1 protein was expressed in COS7 cells, a correspondingly larger protein and some degradation protein were detected only by the anti-Myc antibody (Fig. 2B; lane 1) for the positive control. The protein size detected in this experiment was as previously expected. These results showed that the gene of this $S I R P-B 2$ surely had a coding potential, as expected.

To determine the chromosomal localization of the SIRP$B 2$ gene, PCR-based monochromosomal somatic cell hybrid mapping (Quantum, Montreal, Quebec, Canada) was performed with a set of $3^{\prime}$ untranslated region (UTR) primers. This study indicated that the SIRP-B2 gene is located on human chromosome 20 (Fig. 3A). To further refine the subchromosomal location of the gene, radiation hybrid (RH) panels (Stanford G3 and Genebridge 4) were utilized. Both of the linkage analyses of the PCR results (data vec- 
tors for SIRP-B2 of the Stanford G3 and the Genebridge 4 RH panels: 10000100000000001010100000010000010 110000000000000100010000100000100000000100000 010 and 1001001001010000001010111111110101010001 111011010000001110000001011000010000010000100 10000001 , respectively) showed that the $S I R P-B 2$ gene was linked to several markers in the distal region of chromosome 20p13 near the marker D20S906, with lod score values higher than 10.8. The most likely order of the refined loci is shown in Fig. 3B. The SIRP-B2 gene is between SHGC56794 and SHGC-21110, and is adjacent to the marker D20S906. It had been reported that the SHPS-1 gene is localized to chromosome 20p13 near SIRP-B2 (Yamao et al. 1997; Eckert et al. 1997). Other genes that have been mapped to chromosome 20p13 include the M2-acute myeloblastic leukemia (AML) translocation (Xue et al. 1997) and acute promyelocytic leukemia (APL) translocation genes (Yamamoto et al. 1998). Furthermore, the gene that, presumptively, causes Hallervorden-Spatz syndrome was also reported to be mapped to chromosome 20p12.3-13 (Taylor et al. 1996). Our precise chromosomal positioning data should contribute toward positional candidate approaches for these disease genes linked to this locus. Future studies will address the biological role of SIRP-B2 in this regard.

Acknowledgments This work was supported in part by a Grant-in-Aid for Scientific Research on Priority Areas and for COE Research from the Ministry of Education, Science, and Culture of Japan, and by a Grant under the Monbusho International Scientific Research Program. We thank Yu-ki Iwata for her excellent technical assistance.

\section{References}

Dietrich J, Cella M, Seiffert M, Bufring H-J, Colonna M (2000) Cutting edge: signal-regulatory protein b1 is a DAP12-associated activating receptor expressed in myeloid cells. J Immunol 164:9-12

Eckert C, Olinsky S, Cummins J, Stephan D, Narayanan V (1997) Mapping of the human P84 gene to the subtelomeric region of chromosome 20p. Somat Cell Mol Genet 23:297-301

Fujioka Y, Matozaki T, Noguchi T, Iwamatsu A, Yamao T, Takahashi N, Tsuda M, Takada T, Kasuga M (1996) A novel membrane glycoprotein, SHPS-1, that binds the SH2-domain-containing protein tyrosine phosphatase SHP-2 in response to mitogens and cell adhesion. Mol Cell Biol 16:6887-6899

Kharitonenkov A, Chen Z, Sures I, Wang H, Schilling J, Ullrich A (1997) A family of proteins that inhibit signalling through tyrosine kinase receptors. Nature 386:181-186
Kim SO, Jiang J, Yi W, Feng GS, Frank SJ (1998) Involvement of the Src homology 2-containing tyrosine phosphatase SHP-2 in growth hormone signaling. J Biol Chem 273:2344-2354

Kozak M (1989) The scanning model for translation: an update. J Cell Biol 108:229-241

Kuramochi S, Matsuda S, Matsuda Y, Saitoh T, Ohsugi M, Yamamoto $\mathrm{T}$ (1996) Molecular cloning and characterization of Byp, a murine receptor-type tyrosine phosphatase similar to human DEP-1. FEBS Lett 378:7-14

Machida K, Matsuda S, Yamaki K, Senga T, Thant AA, Kurata H, Miyazaki K, Hayashi K, Okuda T, Kitamura T, Hayakawa T, Hamaguchi M (2000) v-Src suppresses SHPS-1 expression via the Ras-MAP kinase pathway to promote the oncogenic growth of cells. Oncogene 19:1710-1718

Matsuda S, K-Tsuzuku J, Ohsugi M, Yoshida M, Emi M, Nakamura Y, Onda M, Yoshida Y, Nishiyama A, Yamamoto T (1996) Tob, a novel protein that interacts with p185erbB2, is associated with antiproliferative activity. Oncogene 12:705-713

Matsuda S, Miyazaki K, Ichigotani Y, Kurata H, Takenouchi Y, Yamamoto T, Nimura Y, Irimura T, Nakatsugawa S, Hamaguchi M (2000) Molecular cloning and characterization of a novel human gene (NESCA) which encodes a putative adapter protein containing SH3. Biochim Biophys Acta 1491:321-326

Saginario C, Sterling H, Beckers C, Kobayashi R, Solimena M, Ullu E, Vignery A (1998) MFR, a putative receptor mediating the fusion of macrophages. Mol Cell Biol 18:6213-6223

Sano S, Ohnishi H, Omori A, Hasegawa J, Kubota M (1997) BIT, an immune antigen receptor-like molecule in the brain. FEBS Lett 411:327-334

Sano S, Ohnishi H, Kubota M (1999) Gene structure of mouse BIT/ SHPS-1. Biochem J 344:667-675

Seki N, Hattori A, Hayashi A, Kozuma S, Ohira M, Hori T, Saito T (1999) Structure, expression profile and chromosomal location of an isolog of DNA-PKcs interacting protein (KIP) gene. Biochim Biophys Acta 1444:143-147

Taylor TD, Litt M, Kramer P, Pandolfo M, Angelini L, Nardocci N, Davis S, Pineda M, Hattori H, Flett PJ, Cilio MR, Bertini E, Hayflick SJ (1996) Homozygosity mapping of Hallervorden-Spatz syndrome to chromosome 20p12.3-p13. Nat Genet 14:479-481

Ueki N, Seki N, Yano K, Masuho Y, Saito T, Muramatsu M (1999) Isolation and characterization of a novel human gene (HFB30) which encodes a protein with a RING finger motif. Biochim Biophys Acta 1445:232-236

Veillette A, Thibaudeau E, Latour S (1998) High expression of inhibitory receptor SHPS-1 and its association with protein-tyrosine phosphatase SHP-1 in macrophages. J Biol Chem 273:22719-22728

Xue Y, Yu F, Xin Y, Lu D, Zou Z, Guo Y, Xie X (1997) t(8;20) (q22;p13): a novel variant translocation of $\mathrm{t}(8 ; 21)$ in acute myeloblastic leukaemia. Br J Haematol 98:733-735

Yamamoto K, Hamaguchi H, Nagata K, Kobayashi M, Takashima T, Taniwaki M (1998) A new complex translocation $(15 ; 20 ; 17)$ (q22;p13;q21) in acute promyelocytic leukemia. Cancer Genet Cytogenet 101:89-94

Yamao T, Matozaki T, Amano K, Matsuda Y, Takahashi N, Ochi F, Fujioka Y, Kasuga M (1997) Mouse and human SHPS-1: molecular cloning of cDNAs and chromosomal localization of genes. Biochem Biophys Res Commun 231:61-67 